\title{
Chile: Expansión Del Cultivo E Industria Del Salmón Y Agricultura Familiar Campesina
}

\author{
(nueva ruralidad y persistencia campesina).
}

Sergio Gómez E.

\section{Resumen}

Se presentan antecedentes sobre la persistencia por mantener la condición de campesinos en espacios donde se consolida una situación de nueva ruralidad. Para ello, se analizan datos de una reciente investigación, donde se analizó el impacto de la expansión de la salmonicultura en una zona con un claro predominio de economía campesina en el sur de Chile ${ }^{132}$.

Uno de los aspectos que llamó la atención en los resultados de este estudio, es la simultaneidad que se observa entre:

- un proceso de proletarización de los hijos de las familias campesinas que se incorporan al trabajo de las empresas salmoneras;

- la permanencia de estas familias en espacios rurales, y

- las relaciones de interdependencia entre las familias "independientes" que forman los jóvenes y la economía campesina de los padres, quienes no se incorporan a estas nuevas actividades.

El trabajo pretende enriquecer la discusión del tema sobre la nueva ruralidad en la medida que entrega antecedentes sobre un aspecto central de el, la participación familiar en actividades no agrícolas. Ello no significa que los miembros de la familia dejen de ser parte de la economía familiar campesina, sino que se generan nuevas relaciones en el contexto de una comunidad campesina ampliada con múltiples empleos e ingresos.

\footnotetext{
* Profesor Universidad Austral de Valdivia.

${ }^{132}$ Industria del Salmón y Agricultura Familiar en Chiloé. Sergio Gomez E., en conjunto con Jorge Echenique, Carlos Amtmann y Rodrigo Rolando. Universidad Austral de Chile - ODEPA, Valdivia/Santiago, diciembre 2001.
} 


\section{I.- Nueva Ruralidad.}

En esta oportunidad no se va a reseñar la bibliografía sobre el tema ${ }^{133}$ sino que se presenta un breve contrapunto de este concepto, con la visión tradicional de lo rural, para luego destacar sus principales rasgos.

A nuestro juicio, la visión tradicional de la ruralidad, corresponde a la mirada que de ella tenían los técnicos y profesionales ligados a las disciplinas agronómicas. Lo rural se hacía equivalente al espacio donde se realizaban actividades silvoagropecuarias. Además, se reconocía que se trataba de poblaciones relativamente aisladas con una baja densidad demográfica y con indicadores que demostraba una situación de atraso generalizado. Esta situación era socialmente subvalorada y explicaba, en parte, la migración desde el campo hacia centros urbanos, donde estas poblaciones podrían alcanzar situaciones de bienestar.

Para la concepción moderna de la ruralidad, se trata de un espacio donde se establece un tipo especial de relaciones sociales, básicamente personales, las que se pueden realizar en la medida que los miembros de estos espacios rurales permiten establecerlas y ello puede ocurrir cuando se pueden dar condiciones de prolongada vecindad y complejas relaciones familiares entre sus habitantes. Entonces, el tema de la baja densidad poblacional es una condición, para que puedan existir verdaderas comunidades en los espacios rurales.

Ahora bien, la otra diferencia fundamental de esta nueva concepción de la ruralidad, es el hecho de que en este espacio se desarrolla un conjunto de actividades, además de la agricultura, como es la minería, la pesca, la industrias medianas y pequeñas, los establecimientos dedicados a las reparaciones, la artesanía, el turismo, la extracción de recursos naturales, el comercio, el transporte, etc. A su vez, en estos espacios se realizan toda una gama de servicios sociales, como es la educación, la salud, la seguridad, la vivienda, el deporte, la vida comunitaria, actividades religiosas, etc.

Entonces, la nueva ruralidad se encuentra en estos espacios, donde se realiza todo este tipo de actividades productivas, de servicios y sociales y se establecen relaciones de carácter personal entre sus miembros.

A modo de resumen, se puede recurrir a las siguientes imágenes:

- mientras para la concepción de la ruralidad tradicional la población rural coincidía con la actividad agrícola;

- en la versión moderna de ruralidad, su población se hace más bien asimilable con la condición de ser "provinciana."

En el primer caso, primaba una aproximación hacia una actividad ("trabajaban en el campo"); en el segundo, se privilegia un tipo de relación social ("todos nos conocemos").

\footnotetext{
${ }^{133}$ Ver Sergio Gómez E. La Nueva Ruralidad: ¿Qué tan nueva?, Universidad Austral de Chile - Ediciones LOM, Santiago 2002.
} 
Ello, por cierto, va más allá de los estrechos criterios demográficos y censales, y plantea que los espacios rurales establecen sus límites hasta donde este tipo particular de relación social puede alcanzar. De paso, rechaza una visión dicotómica entre lo urbano y lo rural y más bien plantea una noción de gradualidad, en donde lo rural termina y comienza lo urbano cuando este tipo de relación social que se ha definido se extingue.

Recientemente he propuesto considerar situaciones que corresponden a nueva ruralidad, aquellas que abarcan tres dimensiones acumulativas en cuanto a:

1. El tipo de espacio y las actividades que se realizan, tema que ya ha sido abordado.

2. La especificidad que la distingue en cuanto a tipo de relaciones sociales, $y$

3. El alcance que abarca la noción propuesta sobre lo rural. Mas adelante, vamos a retomar estas últimas dos dimensiones.

Además, se observa en la actualidad una alta valoración hacia el espacio rural y la ruralidad y que ya no se la considera como una categoría residual frente a lo moderno y urbano.

En lo que se refiere a su especificidad, es la parte fundamental del argumento. Lo rural comprende un tipo de relaciones sociales con un componente personal que se pueden desarrollar en territorios con una baja densidad de población relativa. Esta relación personal, tiene una fuerte base en las relaciones vecinales, con una prolongada presencia, y de grados de parentesco entre una parte significativa de los habitantes.

Lo rural, en definitiva, a pesar del proceso de globalización en marcha, puede llegar a ser una de las condiciones que permite mantener algunos rasgos de identidad frente a las fuerzas homogéneas que se expresan a través de los medios de comunicación, del consumo, etc.

Por último, en cuanto a su alcance, incluye determinados espacios normalmente considerados como urbanos, como parte integrante de la ruralidad.

Como señala A. Schejtman ${ }^{134}$, normalmente en la literatura se ha destacado el papel de la agricultura en el desarrollo desde el ángulo de lo urbano - industrial y se refiere a la transferencia de recursos del campo a la ciudad (fuerza de trabajo, alimentos, etc.). Pero también se puede plantear la pregunta al revés ¿ cual es el papel que podría tener el núcleo urbano en el desarrollo de su entorno rural? Mejor todavía resulta plantear la necesidad de analizar las articulaciones que se establecen entre lo urbano y lo rural, como condición para entender el funcionamiento de la economía y la política local.

Este mismo tema ha sido tratado cuando se señala que se debe "reexaminar la problemática del desarrollo rural (para) insertarla en el marco del desarrollo de las economías locales, es decir enfatizar las articulaciones entre el núcleo urbano y su hinterland agrícola, analizando como se entrelazan los distintos mercados y como inciden sobre su funcionamiento los determinantes externos. En este marco pueden crearse espacios

\footnotetext{
${ }^{134}$ Ver: Alejandro Schejtman. Las dimensiones urbanas en el desarrollo rural. Revista de la Cepal $\mathrm{N}^{\mathrm{o}}$ 676, Santiago, abril de 1999.
} 
de participación sustantiva y plantearse tanto los temas de transformación productiva, como los de pobreza y medio ambiente".

En cuanto al alcance, es necesario considerar dos dimensiones.

(i) El alcance que tiene este tipo de relaciones en concentraciones urbanas, y

(ii) El grado de integración a los servicios, mercados, etc.

Con respecto a lo primero, el tipo de relaciones sociales que se desarrolló en el punto anterior, desde un eje que puede ser considerado como su punto focal, se extiende hacia espacios más densamente poblados, hasta que se extingue la relación personal y pasan a primar las relaciones funcionales.

El tipo de relación social que caracteriza la ruralidad depende, en un grado importante del grado de articulación entre los habitantes rurales tradicionalmente considerados como tales y los habitantes de concentraciones urbanas, depende de los grados de integración que exista entre estas concentraciones urbanas y su entorno rural.

A su vez, diversos autores ${ }^{135}$, sostienen que una característica de esta situación de nueva ruralidad, la actividad agropecuaria, irá perdiendo importancia dentro de los espacios rurales. A su vez, se supone que existiría una creciente perdida de importancia de la agricultura familiar campesina en estos espacios.

Si bien lo primero puede ser cierto, en este trabajo se argumenta que la importancia de la agricultura campesina puede llegar a ser determinante para viabilizar las nuevas actividades productivas modernas, que se desarrollan en los espacios de nueva ruralidad.

Esta hipótesis se pretende demostrar en este trabajo en caso de la expansión del cultivo e industria del salmón en el contexto rural de Chiloé.

\section{II.- Agricultura campesina en Chiloé}

La realidad de la agricultura campesina en Chiloé es ampliamente conocida en los medios especializados, por lo que solo se presentará una apretada reseña de sus principales características.

La Provincia de Chiloé corresponde a un archipiélago en el Sur de Chile con una fuerte presencia de explotaciones campesinas estimándose un número superior a los 12.000 pequeños agricultores, concentrados mayoritariamente en la zona sur oriental de la isla.

\footnotetext{
${ }^{135}$ Ver Jorge Echenique. Análisis prospectivo de la agricultura chilena. En: La agricultura chilena del 2010. Tres Visiones Sociopolíticas. ODEPA, Santiago, 2000.

Julio Berdegué, Eduardo Ramírez, Ximena Milicecic, Tomas Reardon y Germán Escobar. Empleo e Ingreso Rural en Chile. BID, CEPAL, FAO y RIMISP, Santiago, 1999.
} 
A su vez, la estructura de tenencia en Chiloé es diferente a la de su contexto regional cercano, teniendo más semejanzas con la agricultura de secano del Chile Central. En cuanto con referencia al origen de la propiedad rural, en Chiloé se entregaron mercedes de tierras, pero en superficies notablemente inferiores que en la zona central.

Además, la colonización extranjera del Siglo XIX, tan importante en el resto del sur, tiene menor importancia en Chiloé.

Por otra parte, en general, la presencia de empresas agropecuarias es menor que en otras regiones de Chile y, por lo tanto, también es reducida la presencia de fuerza de trabajo asalariada.

En Chiloé la pequeña propiedad y el minifundio han sido tradicionalmente predominantes. Esta realidad llevó a que Pérez Rosales, promotor de la colonización Europea del Siglo XIX, escribiera en 1859 "todo chilote es propietario" y que Mac Bride, un agudo observador del campo chileno a mediados del Siglo XX, en su clásico estudio titulado "Chile su Gente y su Tierra", observa que de las 7.000 propiedades existentes en la isla, 6.300 de ellos tengan menos de 20 hás." ${ }^{136}$

En la actualidad si se clasifican las propiedades agrícolas de Chiloé de acuerdo a tamaño, se puede observar una coincidencia con su ubicación geográfica. Por otro lado, las propiedades grandes, predios que ocupan más de 200 has., se encuentran ubicadas en la costa occidental y se han dedicado mayoritariamente a la explotación forestal. A su vez, los predios medianos se ubican en la parte central de la isla con superficies entre las $50 \mathrm{y}$ las 200 has., dedicándose fundamentalmente a la ganadería y a la explotación forestal.

Los predios con predominio de agricultura familiar campesina se encuentran en áreas cercanas al mar interior, donde se ubican los centros de cultivo del salmón.

\section{III.- Expansión del cultivo y la industria del salmón en Chiloé.}

Sobre este aspecto hay que destacar la importancia y el dinamismo de la salmonicultura en las últimas décadas en Chiloé y en la Región. Lo anterior se refleja en la presencia de fuertes inversiones de capitales nacionales y transnacionales, con un rápido crecimiento de la producción. El sector pesquero, constituido por la pesca extractiva y la acuicultura el año 2000 llegó a exportar US 1.784 millones. Al respecto, la acuicultura generó US 1.050.millones, lo que representa un $56 \%$ del sector pesquero.

Junto a las frutas, la madera y el vino, el salmón es uno de los principales productos no tradicionales que Chile exporta. Con una expansión rápida y sostenida en 20 años ha alcanzado el $25 \%$ del mercado mundial. Ello lo sitúa como el segundo exportador mundial de salmones, después de Noruega.

${ }^{136}$ Jorge M Mc Bride. Chile su Tierra y su Gente. ICIRA, Santiago, 1973. 
En 1999, Chile exportó productos de salmón por un valor de US\$ 818 millones, cifra que se elevó a US\$ 973 millones durante el año 2000.

El salmón ha llegado a sobrepasar el 50\% de las exportaciones pesqueras, manteniendo un sostenido ritmo de crecimiento a lo largo de los años y con un fuerte impacto en el empleo. Las empresas salmoneras ocupan 24.800 empleos de forma directa y 12.000 en forma indirecta. El salmón en sus diferentes estados de desarrollo y de producción es trasladado 6 veces de lugar antes de convertirse en un producto final.

La fuerte expansión de esta actividad entró en crisis en el año 2002, por la baja de los precios en el mercado mundial debido al crecimiento de la oferta chilena. Ello también ha motivado interferencias para la entrada del salmón chileno a la UE debido a la acusación de prácticas comerciales ilícitas (dumping)

\section{IV.- Salmonicultura y la economía campesina en Chiloé.}

El desarrollo del cultivo y de la industria del salmón en la isla de Chiloé, ha afectado de manera importante la vida de los habitantes de la isla y particularmente la situación de las familias campesinas.

Esta industria muestra una alta demanda de empleo la cual ha penetrado el sector rural, disminuyendo notoriamente las migraciones que formaban parte de la situación habitual de los jóvenes en las familias en Chiloé en décadas pasadas. Sin embargo, esta situación no ha alcanzado a tener impacto suficiente como para revertir las migraciones preexistentes y generar migraciones de retorno, como se analizará más adelante.

Entre las familias campesinas que permanecen en la isla, predomina la percepción de una situación de mayor progreso y bienestar que se observa actualmente y que se ha visto favorecida con mejoras en las condiciones de vida, mayores accesos, caminos y el establecimiento de un comercio más amplio. Lo anterior, en particular, se asocia a una mejoría en las posibilidades que tienen los jóvenes de obtener empleo, sin tener que abandonar la isla, ni menos, el medio rural al que pertenecen.

A pesar de la influencia de esta industria en la isla, las familias siguen manteniendo su condición de rurales, en la medida que el trabajo de algunos de sus integrantes no impide que tanto la actividad agrícola, en la medida que refiere fundamentalmente a actividades de huerto, como la ganadera, la cual es de estructura muy simple y precaria, sigan su curso habitual. La principal conclusión del estudio es que la actividad salmonera no produce una disminución de este tipo de actividades y, por ende, no tiene efectos significativos sobre la estructura productiva agropecuaria de las unidades familiares campesinas. 
Esta situación, se explica fundamentalmente a partir de la funcionalidad que tiene la producción de la parcela destinada al autoconsumo, la cual sigue resultando fundamental para bajar el costo de la alimentación de los trabajadores rurales hijos de los campesinos ya adultos que trabajan en la salmonicultura. De esta forma, se puede constatar que las actividades agrícolas orientadas al autoconsumo no requieren del trabajo directo y exclusivo del jefe de hogar o de quien trabaja en la salmonera, los cuales son reemplazados sin mayores dificultades al interior de la propia familia involucrada, por lo que, en definitiva, dicha actividad, no ha sido afectada significativamente. A su vez, la presencia y mantención de este tipo de actividad agrícola explica, en parte importante, el hecho de que los niveles de ingreso se mantengan en el nivel del salario mínimo fijado por ley (aprox. US $\$ 150 .-$ por mes)

En este sentido, el rol que juega la agricultura campesina, se explica por las siguientes razones. En primer lugar, los productos tradicionales de la isla no tienen un precio que resulte atractivo para su comercialización, por lo que no existe el incentivo para propender a una agricultura intensiva. En segundo lugar, hay que agregar la escasez de fuerza de trabajo familiar, en la medida que se encuentran trabajando en las salmoneras, se debe agregar la dificultad de encontrar trabajadores agrícolas para contratar, y si lo logran es a un precio muy elevado, aspecto que también afecta la posibilidad del trabajo agrícola intensivo.

Todo lo anterior explica que la principal valoración de la actividad vinculada a la salmonicultura de parte de las familias campesinas de Chiloé, es el hecho de que dichas familias pueden contar con fuentes de trabajo que ofrecen ingresos estables, aun cuando dicho ingreso, en la mayoría de los casos, corresponde como se ha planteado al salario mínimo.

No obstante lo anterior, este bajo ingreso resulta atractivo para el campesino, en la medida que se den las siguientes condiciones: Por un lado, el hecho de que gran parte de las familias campesinas disponen de una vivienda en el medio rural $\mathrm{y}$, por lo tanto, no tienen gastos relevantes asociados a ella (arriendo de una vivienda en el pueblo, el pago de una pensión, etc.). Por el otro, el hecho de que una parte importante del costo de su alimentación proviene de la explotación familiar (papas, ovejas, trigo, manzana, etc.) ya sea a través de la existencia de huertos familiares propios o de parte de cosechas provenientes de la explotación del padre o suegro, en su calidad de antiguo jefe de la familia.

Además se pudo identificar las razones de los que no se pudieron incorporar a la industria salmonera y los otros que si lo hicieron: básicamente se refiere a razones de edad. En efecto, sólo se incorporan los jóvenes, entre 21 y 30 años, ocasión en la que se inician en la vida laboral.

Por lo tanto, el corte etáreo que explica la incorporación o exclusión a la industria del salmón es importante. De esta forma, aquellos campesinos y sus familiares que tuvieron la posibilidad de incorporarse como asalariados en las faenas ligadas a la industria del salmón, lo hicieron. Por el contrario, aquellas personas que tuvieron algún impedimento físico o una edad avanzada, no se incorporaron. 
El corte etáreo también se explica por la mayor capacidad de adaptación que tienen los jóvenes a situaciones laborales propias de una lógica industrial con todas las implicancias que ello conlleva en el plano de la innovación, del adiestramiento y de la disciplina laboral.

A partir de lo anterior, cabe señalar que la incorporación masiva de jóvenes provenientes de familias campesinas a la industria salmonera tiene varias consecuencias, entre las cuales cabe destacar que existe una fuerte disminución de las migraciones en los jóvenes, fenómeno tradicional en la isla en décadas pasadas. A pesar de lo anterior, no se observan migraciones de retorno, ya que los ingresos que se ofrecen no serían atractivos para los migrantes ya que tendrían una situación estabilizada en el lugar de destino.

Por su parte, la explotación agrícola de las familias que tienen miembros trabajando en las salmoneras, tiene un sentido de apoyo al consumo de toda la familia, incluso más allá de la conformación del hogar.

Los mayores ingresos provenientes del cultivo y de la industria del salmón, se dedican básicamente a mejorar las condiciones de vida de los hogares. Lo anterior se expresa en el mejoramiento de las condiciones de la vivienda, en mayor equipamiento para el hogar, en mejores condiciones de vestuario y alimentación. Así también, este mayor ingreso les ha permitido disponer, en muchos casos, de medios de transporte propios como son : autos, camionetas y motos.

Así, los ingresos provenientes de la industria salmonera, no se destina para mejorar la capacidad productiva de las unidades campesinas, salvo en los casos de una mantención mínima de las explotaciones (abono para las siembras destinadas al autoconsumo, reparar cercos deteriorados, etc.).

En este sentido, si bien la salmonicultura no ha afectado la estructura productiva de las familias, si se ha podido constatar que ha sido afectada la situación de empleo y del ingreso. Las condiciones de vida que tienen las familias campesinas que tienen componentes trabajando en las salmoneras es muy superior a la que existía en el pasado.

Se puede observar una diferencia sustancial respecto al nivel de ingreso en favor de quienes se incorporaron a la actividad salmonera. En efecto, la mayoría de las familias ubicadas en áreas de agricultura campesina que tienen al menos un integrante trabajando actualmente en una salmonera tienen un ingreso percápita promedio de aproximadamente $\$ 63.000$. Por el contrario, la mayoría de las familias que no se incorporó a esta actividad, es decir que no tienen un integrante trabajando en la industria salmonera tienen un ingreso percápita promedio de aproximadamente $\$ 50.000$.

De esta forma, el hecho de disponer de un ingreso periódico asegurado y con ello mejorar las condiciones de vida y de trabajo, actúan como elementos que frenan las altas migraciones que caracterizaron a los familiares de las unidades campesinas de Chiloé. Los antecedentes del Censo de Población del año 2002 confirman este hecho. Mientras la tasa de crecimiento intercensal a nivel nacional es de $2 \%$, en la Provincia de Chiloé, el 
crecimiento alcanza al 17,9\%, cifra que se eleva en las comunas donde se concentra la actividad salmonera (Quellón 42,4 \%, Dalcahue 37,4 \% y Castro 30,5 \%)

Sin embargo, el hecho de que la mayoría cuente con un salario mínimo y las condiciones de aislamiento - considerando todos los adelantos logrados - no alcanzan a constituir un foco de atracción para llegar a provocar migraciones de retorno de chilotes adultos que se establecieron en el sur de Argentina o en Punta Arenas. Ello, además se puede explicar por las propias condiciones que han logrado consolidar los antiguos migrantes en sus lugares de destino

En cuanto al destino de los nuevos ingresos de las familias campesinas que se incorporaron a la actividad salmonera, no se han destinado a mejorar las condiciones productivas, por ejemplo, a través de la implementación de maquinaria agrícola, introducción de nuevos cultivos con tecnologías sofisticadas, etc. En cambio, se han destinado a un mayor acceso a bienes de consumo durables, a mejoramiento de condiciones del hogar, instalaciones para el suministro de electricidad, vehículos, vestuario, alimentación, etc.

Otro de los efectos de la llegada de la salmonera a la isla ha sido el cambio en la constitución de la familia y sus tipos de vinculación. En este sentido cabe destacar que, en forma anticipada en el tiempo y con una mayor frecuencia que en décadas anteriores, se generan nuevas familias de jóvenes, con ingresos propios, que apartan casa y que mantienen vínculos con la familia paterna.

En este sentido, se puede constatar una disminución en el tamaño de las familias, a través de un cambio de un tipo de familia tradicional campesino (numerosa y con hijos grandes, todos trabajando en el campo), a una de índole más urbano, es decir, constituida por un matrimonio con dos hijos, relativamente jóvenes (los cuales constituyen una nueva familia).

De esta forma, es posible apreciar la existencia de efectos colaterales, tales como el menor trabajo en actividades agrícolas intensivas o de mayor escala, no así el trabajo en pequeñas superficies, lo que resulta un aspecto fundamental para la mantención de una agricultura orientada al autoconsumo.

Por otra parte, también es destacable la incorporación de la mujer al trabajo de las salmoneras, lo cual genera una situación de mayor autonomía de éstas respecto del hogar, situación que no existe en los casos en que la mujer se incorporaba como fuerza de trabajo familiar.

Este cambio, tiene un sentido de ruptura con relación a la dependencia que caracterizaba a la mujer rural tradicional, en la medida que estos ingresos le permiten efectuar no sólo un aporte significativo (y nada de marginal) a la familia mejorando con ello la calidad de vida de la familia, sino también le crea condiciones para que pueda constituir un nuevo hogar.

Con relación a los aspectos negativos de la incorporación de la salmonicultura en la isla, las familias frecuentemente mencionan que éstos se dan a partir de la llegada masiva de gente de "afuera", lo cual asocian a un aumento de conductas delictuales, tales como robos, 
asaltos y otras conductas indeseadas. Cabe destacar también, que de parte de las familias, no hay mayores referencias, por ejemplo, a situaciones de contaminación o daño ecológico.

En definitiva, la queja se refiere a la llegada de extraños a lugares de alta ruralidad donde las vecindades prolongadas y los intensos lazos familiares han creado las condiciones para que se establezcan relaciones sociales personales y fuertes sistemas de control social, que no siempre logran adaptar sus sistemas de vida a nuevas condiciones de entorno.

Finalmente, hay que consignar que el estudio analizó el contenido de diferentes programas de desarrollo gubernamentales y no gubernamentales destinados a áreas campesinas donde existiera un impacto de la actividad salmonera. Salvo en el caso de una Escuela Agrícola privada que comenzaba a abrirse a la temática de la acuicultura, el resto de los programas eran muy similares a los que se aplican en otras regiones del país. En otras palabras, los programas dirigidos a campesinos afectados por la situación descrita no difieren de aquellos programas de desarrollo agrícola o rural que se implementan en el resto del país.

\section{V.- Conclusiones: nueva ruralidad y persistencia campesina.}

A diferencia de los que ocurría en situaciones de ruralidad tradicional donde la mayoría de los hijos jóvenes de las familias campesinas migraban hacia las ciudades (Figura 2), en el caso de situaciones caracterizadas como de nueva ruralidad, normalmente, la literatura plantea como procesos paralelos e independientes las siguientes situaciones:

a) El caso de campesinos envejecidos que se mantienen vinculados a la explotación agrícola familiar, y por el otro lado,

b) El caso de hijos de estos, que emprenden actividades rurales no agrícolas en espacios rurales, sin mayor vinculación con la explotación agrícola familiar.

Los antecedentes (Figura 3) presentados muestran, por el contrario, que:

- Campesinos envejecidos pueden seguir dedicados a explotación agrícola, porque tienen hijos que se dedican a otros oficios, les aportan ingresos derivados de esas actividades;

- Campesinos jóvenes se pueden incorporar a estas actividades rurales no agrícolas porque cuentan con un sustento respaldado por la explotación agrícola familiar que puede proveer dos elementos básicos que no vienen del salario de la industria acuícola: el pago de la vivienda y el pago de consumo de bienes alimenticios básicos.

Ello muestra la persistencia del campesinado, como forma de organizar la producción y como forma de vida, que ha logrado sobrevivir a los más diversos sistemas económicos. Surge con el feudalismo, se desarrolla con el sistema capitalista, resistió los avatares del 
socialismo y, al parecer, pretende demostrar que intenta persistir durante el neoliberalismo globalizado.

\section{Figura N 1}

Situación Familia Campesina tradicional Joven

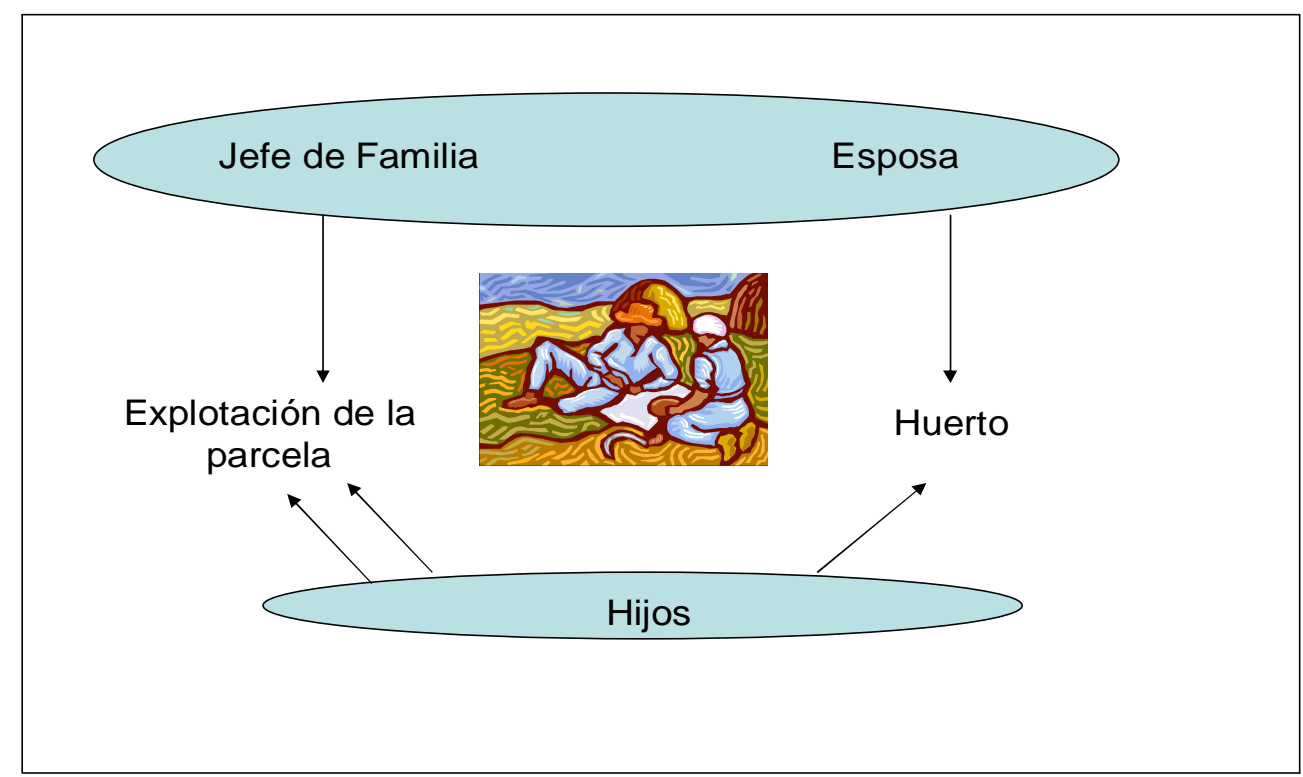

Figura N 2 
Situación Familia Campesina adulta tradicional

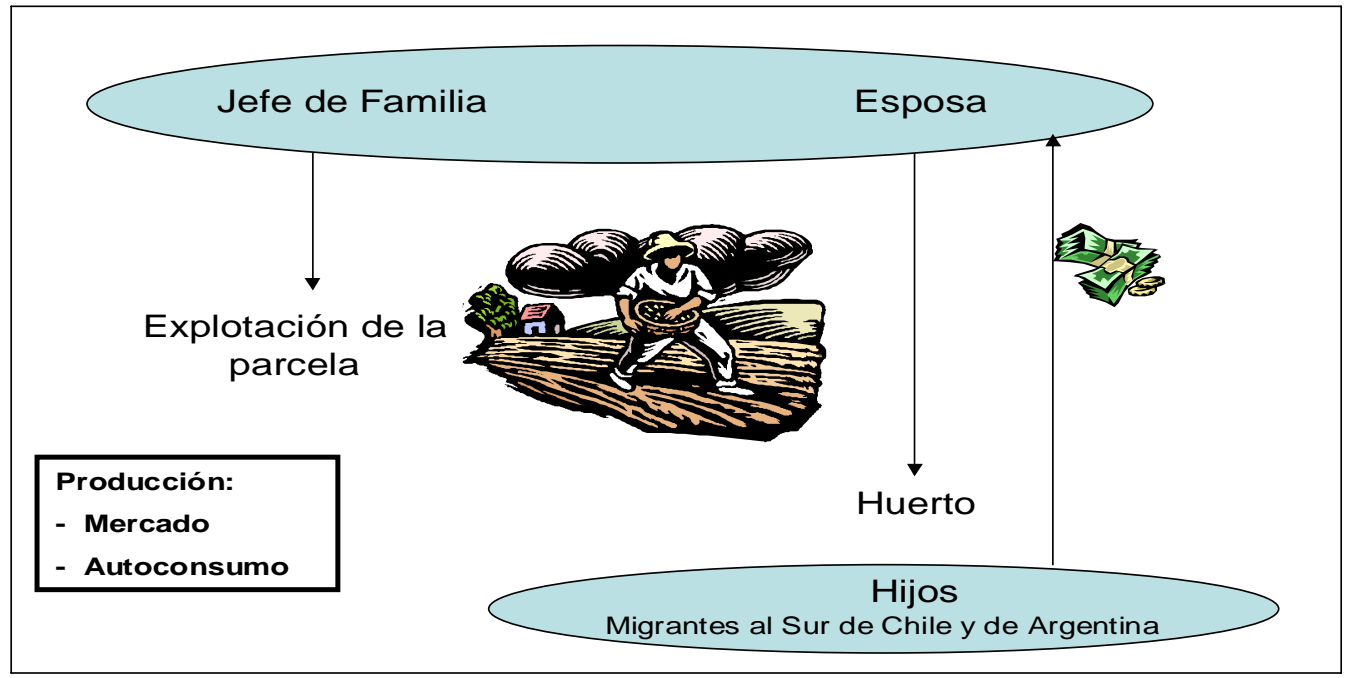

Figura N 3

Situación Familia Campesina adulta nueva ruralidad

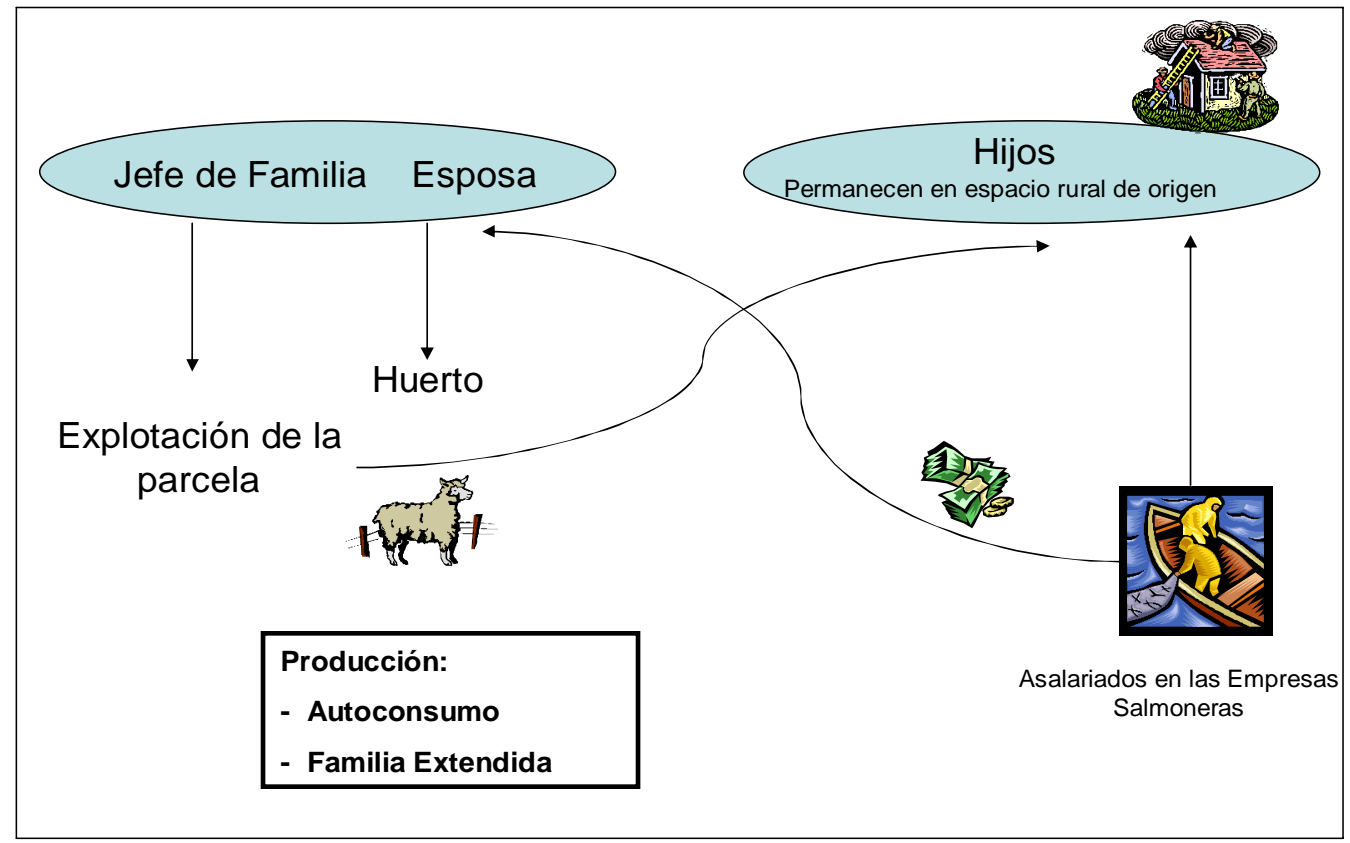


Es importante resaltar que en esta situación, donde la vivienda y parte importante de la alimentación de los jóvenes hijos de campesinos provienen de la explotación familiar, viabiliza la expansión de la industria acuícola, al permitir que los salarios de estos trabajadores jóvenes se mantengan en el nivel del salario mínimo legal. Si estos gastos tuvieran que ser desembolsados por estas familias, difícilmente tendrían interés en incorporarse a esta actividad.

Ello explica la ventaja comparativa que se reconoce en las empresas salmoneras que se encuentran en Chile, cuando se resalta el bajo costo de la fuerza de trabajo ${ }^{137}$.

El tema de la persistencia campesina ha sido una reflexión recurrente en la sociología rural. Desde los aportes de los clásicos, particularmente las teorías sobre economía y diferenciación campesinas se ha resaltado la capacidad de adaptación y de persistencia que ha tenido a lo largo del tiempo.

Incluso, algunos de los cambios más significativos que ocurrieron en el Siglo XX expresan este hecho. Por una parte, las dificultades que tuvieron los países que intentaron construir el socialismo, terminando con los campesinos, enfrentaron en el campo, severos problemas. Por otra parte, la activa participación campesina en la revolución Mexicana, ha sido analizada como una expresión límite de sobrevivencia de los campesinos. Arturo Warman en trabajo clásico ${ }^{138}$, plantea que su estudio sobre las movilizaciones en el Estado de Morelos - cuna rural de la revolución mexicana - que él estudia, es una narración de la persistencia de estos campesinos a través de una historia cotidiana, carente de dramatismo y retórica: lo que les interesó fue sobrevivir manteniendo su condición de campesinos.

\footnotetext{
137 “'”Para ello, la Región de los Lagos (X) en particular tiene ventajas comparativas naturales y mano de obra semi - calificada abundante con salarios bajos en comparación con la competencia lo que le ha permitido a Chile incursionar muy exitosamente en él". Sergio Gomez E., en conjunto con Jorge Echenique, Carlos Amtmann y Rodrigo Rolando. Industria del Salmón y Agricultura Familiar en Chiloé. Universidad Austral de Chile - ODEPA, Valdivia/Santiago, diciembre 2001. Basado en:

- Martine Dirven Los encadenamientos de la agricultura chilena vistos en el territorio. -acercamientos a una medición- Proyecto CEPAL/INDAP CHI/00/048: Elementos para el diseño de una propuesta para la superación de la pobreza. División de Desarrollo Productivo y Empresarial CEPAL, Naciones Unidas.

- Cecilia Montero, Claudio Maggi y Constanza Parra. La Industria del Salmón en la X Región: Un cluster globalizado. CEPAL s/f

- Torben Petersen, Gerente de Alimentos, Compañía Pesquera Camanchaca Tendencias de la industria productora y procesadora de salmones en Chile. En Revista Aqua N ${ }^{\circ}$ 58, año 12, noviembre 2000.

- Corporación Terra Australis Fundación Terra. La Insuficiencia de la Salmonicultura en Chile. Aspectos Sociales, Económicos y Ambientales. RPP., Informe N ${ }^{o}$ 1, Santiago, Invierno 2000.

- $\quad$ Fundación Chile. La Salmonicultura en Chile, Santiago,1989.

- Revista Aqua. Noticias Internacional. Fundación Chile, varios ejemplares

- $\quad$ Aqua Noticias. Compendio de la Acuicultura y Pesca en Chile. Directorio 2002, Santiago, 2002.
}

\footnotetext{
${ }^{138}$ Arturo Warman. ...Y venimos a contradecir. Los campesinos de Morelos y el estado nacional. Ediciones La Casa Chata, México, 1976.
} 


\section{Bibliografía}

Aqua Noticias. Compendio de la Acuicultura y Pesca en Chile. Directorio 2002, Santiago, 2002.

Julio Berdegué, Eduardo Ramírez, Ximena Milicecic, Tomas Reardon y Germán Escobar. Empleo e Ingreso Rural en Chile. BID, CEPAL, FAO y RIMISP, Santiago, 1999.

Corporación Terra Australis Fundación Terra. La Insuficiencia de la Salmonicultura en Chile. Aspectos Sociales, Económicos y Ambientales. RPP., Informe $\mathrm{N}^{\circ}{ }^{\circ} 1$, Santiago, Invierno 2000.

Martine Dirven Los encadenamientos de la agricultura chilena vistos en el territorio. acercamientos a una medición- Proyecto CEPAL/INDAP CHI/00/048: Elementos para el diseño de una propuesta para la superación de la pobreza. División de Desarrollo Productivo y Empresarial CEPAL, Naciones Unidas.

Jorge Echenique. Análisis Prospectivo de la Agricultura Chilena. En La Agricultura Chilena del 2010. Tres visiones sociopolíticas. ODEPA, Santiago, 2000.

Fundación Chile. La Salmonicultura en Chile, Santiago,1989.

Sergio Gomez E., en conjunto con Jorge Echenique, Carlos Amtmann y Rodrigo Rolando. Industria del Salmón y Agricultura Familiar en Chiloé. Universidad Austral de Chile ODEPA, Valdivia/Santiago, diciembre 2001.

Sergio Gómez E.. La Nueva Ruralidad: ¿Qué tan nueva?, Universidad Austral de Chile Ediciones LOM, Santiago 2002.

Jorge M Mc Bride. Chile su Tierra y su Gente. ICIRA, Santiago, 1973.

Cecilia Montero, Claudio Maggi y Constanza Parra. La Industria del Salmón en la X Región: Un cluster globalizado. CEPAL s/f

Torben Petersen, Gerente de Alimentos, Compañía Pesquera Camanchaca Tendencias de la industria productora y procesadora de salmones en Chile. En Revista Aqua $\mathrm{N}^{\circ}$ 58, año 12, noviembre 2000.

Revista Aqua. Noticias Internacional. Fundación Chile, varios ejemplares

Alejandro Schejtman. Las Dimensiones Urbanas en el Desarrollo Rural. Revista de la Cepal $\mathrm{N}^{\mathrm{o}} 676$, Santiago, abril de 1999.

Arturo Warman. ...Y Venimos a Contradecir. Los campesinos de Morelos y el Estado Nacional. Ediciones La Casa Chata, México, 1976. 